\title{
Investigation of Novel Peptide Chiral \\ Selectors Prepared by Solid-Phase \\ Synthesis with a tert-Butoxycarbonyl \\ Amino Acid
}

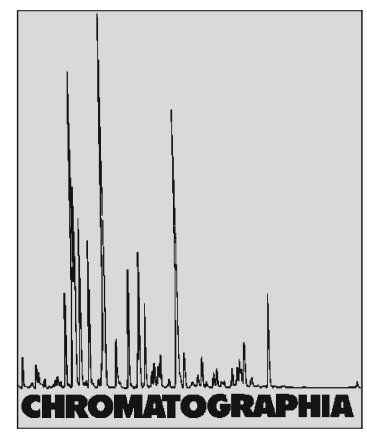

2009, 70, 1501-1504

\author{
Kaname Ohyama ${ }^{1}$, Kana Oyamada ${ }^{1}$, Naoya Kishikawa ${ }^{1}$, Miyuki Arakawa ${ }^{1}$, Yoshihito Ohba ${ }^{2}$, \\ Masahiro Kamino ${ }^{1}$, Mitsuhiro Wada ${ }^{3}$, Kenichiro Nakashima ${ }^{3}$, Naotaka Kuroda ${ }^{1, 凶}$ \\ 1 Department of Environmental and Pharmaceutical Sciences, Graduate School of Biomedical Sciences, Nagasaki University, \\ Nagasaki 852-8521, Japan; E-Mail: n-kuro@nagasaki-u.ac.jp \\ 2 Faculty of Pharmaceutical Sciences, Nagasaki International University, Nagasaki 852-8521, Japan \\ 3 Department of Clinical Pharmacy, Graduate School of Biomedical Sciences, Nagasaki University, Nagasaki 852-8521, Japan
}

\begin{abstract}
A new class of chiral stationary phases (CSP) with peptide chiral selectors was prepared by solid-phase synthesis with a tert-butoxycarbonyl-L-amino acid on silica. The type of amino acid that is favorable for this class of CSP is discussed. Using the CSP with the phenylalanine peptide selector, the effect of peptide length on the enantioselectivity was investigated in normal-phase mode. The applicability of the CSP with a phenylalanine peptide to chiral ligand-exchange chromatography was also examined.
\end{abstract}

\section{Keywords}

Column liquid chromatography

Peptide chiral selectors

Normal-phase separations

Chiral ligand-exchange

Secondary structure

\section{Introduction}

Enantiomers of a chiral drug often have dramatically different pharmacological or toxicological effects and therefore, the separation of enantiomers is very important. LC with a chiral stationary phase (CSP) is a popular method in the field of chiral separation. The design and development of CSPs has attracted much attention for a number of years. To date, CSPs with several chiral selectors, such as cyclodextrins [1], macrocyclic antibiotics [2], polysaccharide derivatives [3], modified amino acids [4, 5], proteins [6] and cinchona alkaloids [7], have been reported and some of them are commercially available. Since CSPs with a protein chiral selector have excellent enantioselectivity, a peptide chiral selector would seem to offer promise. Indeed, research on peptide chiral selectors has progressed [8-12].

In a recent study by us, three CSPs having Phe peptide chiral selectors with different peptide length, $\mathrm{Phe}_{4}, \mathrm{Phe}_{8}$ and $\mathrm{Phe}_{12}$, were individually prepared on aminopropyl silica (APS) by solid-phase synthesis with tert-butoxycarbonyl (Boc) -L-Phe [13]. The highest resolution of model enantiomers $(R / S$ warfarin) was observed for the Phe peptide chiral selector with intermediate length (i.e. $\mathrm{Phe}_{8}$ ), which differed from other peptide chiral selectors for which better resolution was attributable to longer peptide length [9].

In the present study, we evaluated other peptide chiral selectors made from Boc- phenylglycine (Phg), Boc-Val and Boc-Pro in order to find out the kind of amino acid that is most favorable for this class of CSPs. In addition to the previous study using a reversed phase mode [13], the enantioselective investigation of the Phe peptide CSPs having different peptide length were extended to 

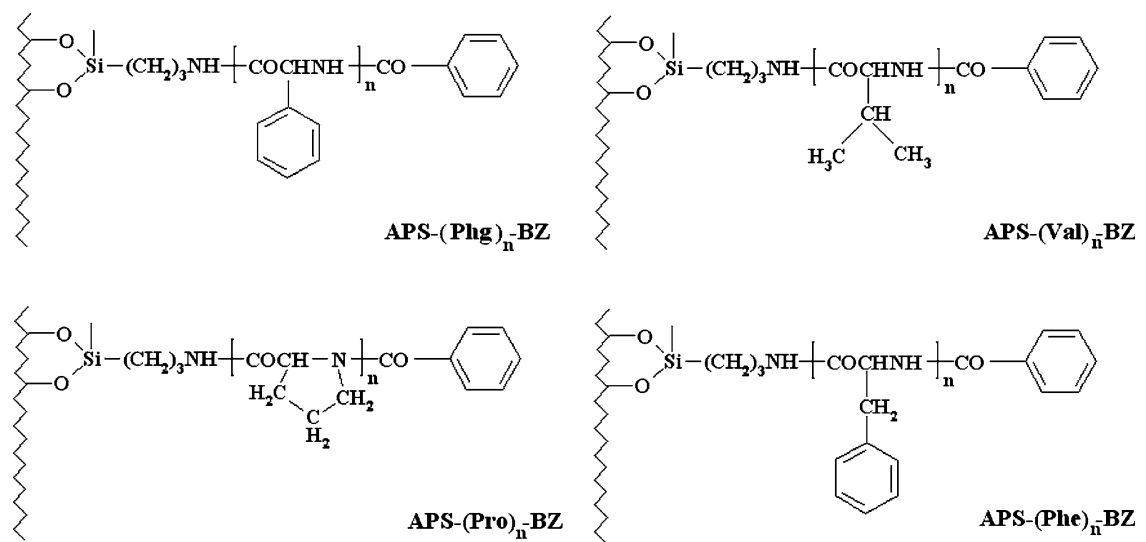

Fig. 1. Structures of the prepared CSPs

normal-phase (NP) mode because the effective chiral recognition in various mobile phase is advantageous because of the different solubilities of the chiral analytes. The applicability of Phe peptide CSPs to chiral ligand-exchange chromatography (CLEC) was also examined.

\section{Experimental}

\section{Chemicals}

Aminopropyl silica (APS, particle size, $5 \mu \mathrm{m}$; pore size, $120 \AA$ ) was a kind gift from Daiso Chemical (Osaka, Japan). Boc-L-Phe, Boc-L-Val and Boc-L-Pro were purchased from the Peptide Institute (Osaka, Japan). Boc-L-Phg was synthesized by use of di-tert-butyl pyrocarbonate as previously reported [14]. Dicyclohexylcarbodiimide (DCC), $N, N^{\prime}$ diisopropylethylamine (DIEA), benzoyl chloride (BZ), triethylamine (TEA), methanol, acetonitrile, hexane, 2-propanol, ninhydrine, mandelic acid, toropic acid, benzoin, amino acids (Phe, Pro, Trp, Tyr and His) and copper (II) sulfate were obtained from Wako Pure Chemicals (Osaka, Japan). Trifluoroacetic acid (TFA) was from Tokyo Chemical Industry (Tokyo, Japan). Dichloromethane (DCM), ethanol, benzyl alcohol, isatin, sodium perchlorate, perchloric acid were from Kishida Chemicals (Osaka, Japan). Warfarin was obtained from Sigma (St. Louis, USA). Optically pure warfarin was prepared from racemic warfarin, using a fractional crystallization method [15].

\section{Solid-Phase Synthesis of the CSPs}

The synthetic procedure of the CSPs with Boc-L-amino acid was as reported in our previous paper [13]. In this procedure, the ninhydrine test was performed to check for comple removal of Boc groups, while the isatin test was performed for terminal prolyl residue of Pro peptides [16]. The modification ratios $\left(\mathrm{mmol} \mathrm{g}^{-1}\right)$ of each peptide on APS were 0.183 for $\mathrm{Phg}$ (elemental analysis [\%]: C, 17.25; H, 1.98; $\mathrm{N}, 2.31$ ), 0.214 for $\mathrm{Val}$ (elemental analysis [\%]: C, 15.26; H, 2.45; N, 2.70), 0.156 for Pro (elemental analysis [\%]: C, 11.39; H, $1.74 ; \mathrm{N}, 1.96)$ and 0.202 for Phe (elemental analysis [\%]: C, 21.25; H, 2.39; $\mathrm{N}, 2.60)$. The ratios were estimated from the value of nitrogen by elemental analysis.

The structures of the CSPs are shown in Fig. 1.

\section{Chromatography}

The prepared CSPs were individually packed into columns (150 $\mathrm{mm} \times$ $4.6 \mathrm{~mm}$ i.d.) by the slurry packing method with methanol [17].

The system for RP-LC consisted of a Waters LC Module 1 (Milford, USA), a Waters HTR-B column oven and a Rikadenki R-01 recorder (Tokyo, Japan) and the one for NP-LC was made up of a Jasco 880-PU pump (Tokyo, Japan), a Jasco 875-UV detector, an Omron E5C3 column oven (Tokyo, Japan) and a Rikadenki NP-2040 recorder (Tokyo, Japan). The system for chiral ligand exchange chromatography (CLEC) included a Hitachi L-7100 (Tokyo, Japan), a Hitachi L-7455 UV-diode array detector and a Hitachi L-7300 column oven. For all the LC systems, flow rate was set at $0.5 \mathrm{~mL} \mathrm{~min}^{-1}$ with detection at $280 \mathrm{~nm}$ and a column oven temperature of $35^{\circ} \mathrm{C}$.

All aqueous solutions were prepared with water that was deionized and distilled using a WG 220 (Yamato Scientific Co, Tokyo) and passed through a water purification system (Puric-Z, Organo Co, Tokyo). The mobile phase was a mixture of $0.5 \mathrm{M}$ aqueous sodium perchlorate and acetonitrile, adjusted with perchloric acid to $\mathrm{pH} 2.0$ for RP-LC and a mixture of $n$-hexane and 2-propanol for NP-LC, while that of CLEC was a mixture of $0.25 \mathrm{mM}$ copper (II) sulfate and acetonitrile. All mobile phases were degassed thoroughly prior to use.

\section{Results and Discussion}

\section{Effect of Amino Acid of the CSPs on the Enantioselectivity}

Warfarin enantiomers were used as a model chiral analytes in order to compare the newly synthesized CSPs, i.e. APS-(Phg) ${ }_{8}$-BZ, APS-(Val) $)_{8}$-BZ and APS-(Pro $)_{8}$-BZ, with APS-(Phe $)_{8}$-BZ under RP-mode. Enantioseparation was achieved on APS-(Val) $)_{8}$-BZ $\left(k_{\mathrm{A}}\right.$, $\left.19.2 ; k_{\mathrm{B}}, 20.1 ; \alpha, 1.05 ; R s, 1.26\right)$ and APS-(Phe) $)_{8}$-BZ $\left(k_{\mathrm{A}}, 27.3 ; k_{\mathrm{B}}, 29.2 ; \alpha\right.$, 1.07; Rs, 1.60) while no chiral resolution was observed on APS-(Phg) $)_{8}$-BZ and APS-(Pro) ${ }_{8}$-BZ. This result suggests that the side chain of the amino acids plays a role in the separation performance of the CSPs. Phg and Phe differ only by the presence of the methylene group between the $\alpha$-carbon and the phenyl group; this methylene group probably gives a high steric flexibility to the interactive site (i.e. the phenyl group) with analytes. In this context, the side chain of Val is also sterically flexible. Pro does not have a 
flexible interaction site and thus, does not appear to be suitable for our proposed CSPs. Besides warfarin enantiomers, the CSPs were used for the enantioseparation of trihexyphenidyl and benzoin which were partially separated on APS-(Phe) $)_{8}$-BZ [13]. However, no chiral separation was observed on the three CSPs. This may be because the side chain of Phe peptide works as a flexible and strongly interactive site in the chiral recognition.

For a peptide chiral selector, the secondary structure introduces more conformational rigidity into the selector and can influence its enantioselectivity. In our previous study [13], we have confirmed that Phe peptide selector exists in an $\alpha$-helical formation. The conformation of the Val peptide is also expected to be an $\alpha$-helix [18]. On the other hand, the Phg peptide does not take an $\alpha$-helical conformation [19] and it is well known that the Pro peptide takes the characteristic conformation of a left-handed polypeptide II helix [20]. These differences of conformation could be partially responsible for their different enantioselectivity.

\section{Effect of Peptide Length in NP Mode}

We evaluated the enantioselectivity of the CSPs in NP-LC mode using four chiral analytes (Table 1). The best resolution was obtained for benzoin enantiomers and as $n$-hexane content increased, the resolution factor increased due to the increase in retention. In RP mode, enantioseparation could not be achieved for mandelic and tropic acids, however partial separation was possible in NP mode. Conversely, warfarin which could be fully separated in RP mode [13] could not be separated in NP mode. The chiral analytes capable of separation with this class of CSPs were changeable by the separation mode used.

Huang et al. [9] reported that a longer peptide selector made of 9-fluorenylmethoxycarbonyl-proline showed a better chiral resolution, whereas in our previous study using RP-LC, the longest Phe peptide selector $\left(\mathrm{Phe}_{12}\right)$ did not demonstrate the best enantioselectivity

Table 1. Chromatographic resolution of chiral analytes in NP mode

\begin{tabular}{|lllll|}
\hline Chiral analyte & $k_{\mathrm{A}}$ & $k_{\mathrm{B}}$ & $\alpha$ & $R_{s}$ \\
\hline Mandelic acid $^{\mathrm{a}}$ & 1.56 & 1.76 & 1.13 & 1.09 \\
Toropic acid $^{\mathrm{a}}$ & 1.41 & 1.50 & 1.06 & 0.74 \\
Warfarin $^{\mathrm{a}}$ & 1.99 & 1.99 & 1.00 & 0 \\
Benzoin $^{\mathrm{b}}$ & 1.51 & 1.74 & 1.15 & 1.44 \\
\hline
\end{tabular}

a APS-(Phe) ${ }_{12}$-BZ, hexane/2-propanol $=80 / 20(v / v)$

${ }^{\mathrm{b}}$ APS-(Phe) $)_{12}$-BZ, hexane/2-propanol $=90 / 10(v / v)$

and the highest resolution was achieved by the $\mathrm{Phe}_{8}$ which has an intermediate peptide length [13]. In contrast to RPLC, the current study showed that only the CSP with the longest peptide selector $\left(\mathrm{Phe}_{12}\right)$ provided an enantioselectivity in NP-LC. This was mainly due to its relatively high hydrophilicity; additionally, the peptide secondary structure produced by the hydrogen bonds facilitated in the organic mobile phase, might contribute to the chiral recognition. The optimum peptide length of our proposed CSPs for enantioseparation should be varied according to the separation mode.

\section{Use of Phe CSP in CLEC}

Due to the good success of derivatized amino acid selectors in enantioseparation via CLEC [21], our proposed CSPs are a good candidate for CLEC in which a chiral selector chelates $\mathrm{Zn}(\mathrm{II}), \mathrm{Ni}(\mathrm{II})$ or $\mathrm{Cu}$ (II) ion through a pair of coordinating electronegative atoms $(\mathrm{N}, \mathrm{O}$ or $\mathrm{S})$.

Four UV-active amino acids, Phe, Trp, Tyr and His, were used as model analytes. As a mobile phase, a mainly aqueous mixture of $0.25 \mathrm{mM} \mathrm{Cu}$ (II) sulfate/acetonitrile $(95 / 5, v / v)$ was used. The tested amino acids were partially enantioseparated in CLEC with APS-(Phe) $)_{8}$-BZ, however no chiral resolution was obtained in RP-LC mode. In this preliminary study, although an absolute enantioseparation was not found because of peak tailing, the values of the separation factors $(\alpha=1.12-$ 1.64) indicated a potential use of our proposed CSP in CLEC.

\section{Conclusion}

A novel class of CSP prepared by solidphase synthesis with a Boc-L-amino acid was characterized. From the comparative study using four amino acid materials, the amino acids having a sterically flexible side chain were possibly preferable for developing this class of CSP in RP-LC. Additionally, the conformation of the peptide selectors may contribute to their enantioselectivity. The CSP with Phe peptide chiral selector was successfully used in both of RP-LC and NP-LC modes. The separations of chiral analytes and the effect of peptide length on chiral recognition were changeable by altering the separation mode. Furthermore, the Phe peptide CSP was potentially applicable to CLEC.

\section{Acknowledgment}

The authors thank Daiso Co., Ltd. (Osaka) for the kind gift of 3-aminopropyl silyl silica gel.

\section{References}

1. Muderawan IW, Ong TT, Ng SC (2006) J Sep Sci 29:1849-1871. doi:10.1002/jssc. 200500491

2. Berthod A, Chen X, Kullman JP, Armstrong DW, Gasparrini F, D'Acquarica I, Villani C, Carotti A (2000) Anal Chem 72:1767-1780. doi:10.1021/ac991004t

3. Yashima E, Yamamoto $\mathrm{C}$, Okamoto $\mathrm{Y}$ (1996) J Am Chem Soc 118:4036-4048. doi:10.1021/ja960050x

4. Danel C, Foulon C, Guelzim A, Park CH, Bonte JP, Vaccher C (2005) Chirality 17:600-607. doi:10.1002/chir.20206

5. Pirkle WH, Finn JM, Schreiner JL, Hamper BC (1981) J Am Chem Soc 103:3964-3966. doi:10.1021/ja00403a076

6. Schill G, Wainer IW, Barkan SA (1986) J Chromatogr 365:73-88. doi:10.1016/ S0021-9673(01)81544-2

7. Lämmerhofer M, Lindner W (1996) J Chromatogr A 741:33-48. doi:10.1016/ 0021-9673(96)00137-9 
8. Dobashi A, Dobashi Y, Kinoshita K, Hara S (1988) Anal Chem 60:1985-1987. doi:10.1021/ac00169a032

9. Huang J, Chen H, Li T (2006) J Chromatogr A 1113:109-115. doi:10.1016/ j.chroma.2006.01.128

10. Shundo A, Sakurai T, Takafuji M, Nagaoka S, Ihara H (2005) J Chromatogr A 1073:169-174. doi:10.1016/j.chroma. 2004.08.062

11. Chen Y, Yu DH (1993) J App Polym Sci 49:851-861. doi:10.1002/app.1993. 070490511

12. Allenmark SG, Andersson S (1994) J Chromatogr A 666:167-179. doi:10.1016/ 0021-9673(94)80379-X
13. Ohyama K, Oyamada K, Kishikawa N, Ohba Y, Wada M, Maki T, Nakashima K, Kuroda N (2008) J Chromatogr A 1208:242-245. doi:10.1016/j.chroma. 2008.08.121

14. Pozdnev VF (1976) Chem Nat Compd 10:782-784

15. West BD, Preis S, Schroeder CH, Link KP (1961) J Am Chem Soc 83:2676-2679. doi:10.1021/ja01473a020

16. Kaiser E, Bossinger CD, Colescott RL, Olsen DB (1980) Anal Chim Acta 118:149-151. doi:10.1016/S0003-2670(01) 93726-2

17. Guan-Sajonz H, Guiochon G (1996) J Chromatogr A 743:247-259. doi:10.1016 0021-9673(96)00300-7
18. Gö M, Hesselink T, Gö N, Scheraga HA (1974) Macromolecule 7:459-467. doi: $10.1021 / \mathrm{ma} 60040 \mathrm{a} 013$

19. Mossel E, Formaggio F, Valle G, Crisma $\mathrm{M}$, Toniolo $\mathrm{C}$, Doi $\mathrm{M}$, Ishida $\mathrm{T}$, Broxterman QB, Kamphuis J (1998) Lett Pept Sci 5:223-225. doi:10.1023/ A:1008809532026

20. Harrington WF, Sela M (1958) Biochim Biophys Acta 27:24-41. doi:10.1016/ 0006-3002(58)90289-0

21. Davankov VA, Navratil JD, Walton HF (eds) (1988) Ligand exchange chromatography. CRC press, Boca Raton 